\title{
Simple approximations for option pricing under mean reversion and stochastic volatility
}

\author{
Christian M. Hafner* \\ Econometric Institute Report EI 2003-20
}

April 2003

\begin{abstract}
This paper provides simple approximations for evaluating option prices and implied volatilities under stochastic volatility. Simple recursive formulae are derived that can easily be implemented in spreadsheets. The traditional random walk assumption, dominating in the analysis of financial markets, is compared with mean reversion which is often more relevant in commodity markets. Deterministic components in the mean and volatility are taken into consideration to allow for seasonality, another frequent aspect of commodity markets. The stochastic volatility is suitably modelled by GARCH. An application to electricity options shows that the choice between a random walk and a mean reversion model can have strong effects for predictions of implied volatilities even if the two models are statistically close to each other.
\end{abstract}

Keywords: Derivatives, stochastic volatility, mean reversion, seasonality, energy markets, spreadsheets

JEL Classification: C14, C22.

${ }^{*}$ Econometric Institute, Erasmus University Rotterdam, P.O. Box 1738, NL-3000 DR Rotterdam, The Netherlands, chafner@few.eur.nl 


\section{Introduction}

Companies active in derivative trading are often faced with the problem of choosing a computational platform that combines simplicity with an adequate representation of the market situation. Unfortunately, the latter is often so complex that highly sophisticated software is needed to generate, for example, forecasts of implied volatilities of option contracts. If the main assumptions of the standard Black and Scholes (1973) model are abandoned, namely constant (or deterministic) volatility and no jumps, closed form solutions are hardly available. In financial and commodity markets, however, empirical work

has widely documented that stochastic volatility and jumps are important features of the data. Although progress has been made in extending option pricing models to these cases, one typically resorts to Monte Carlo simulations. In most practical situations, this may take minutes or hours of computing time, depending on the desired accuracy, and is clearly opposed to a basis for communication within a company. It would be desirable to have a tool that produces results immediately, in order to communicate with traders and be able to use a sensitivity analysis, without neglecting the important features of the market. The computational tool that is used throughout most companies, including traders, is typically a spreadsheet software. Thus, an ideal application would provide results in a spreadsheet with possibilities of interactive use. The advantages of spreadsheets in quantitative finance have been emphasized recently by Aydınlı (2002). Certainly, there are computational issues where spreadsheets are far from being the optimal environment, for example in parameter estimation of complex nonlinear models. But with recent advances in computing technology, it becomes possible to link computationally powerful environments with spreadsheets. An example is the link between Excel and XploRe, termed ReX, as described by Aydınlı, Härdle, Kleinow, and Sofyan (2001).

Options and other derivative contracts were introduced first in financial markets, at about the same time as the appearance of the Black and Scholes (1973) article. From economic theory but also from empirical investigations we know that financial prices such as exchange rates or asset prices can be well described by random walks, see Bachelier (1900) and Chapter 2 of Campbell, Lo, MacKinlay (1997). This explains the predominant use of the standard Black and Scholes formula for options in financial markets. Commodity or energy markets, on the other hand, often show prices that can be assumed to be mean reverting, that is, have some kind of stationary equilibrium level around which prices fluctuate randomly. In some cases, such as interest rates or oil prices, it is typically very difficult to confirm empirically the mean reversion assumption that is made in theories such as Cox, Ingersoll, Ross (1985) for interest rates or Schwartz (1997) for commodity 
markets. In other words, there seems to be a gap between the theoretical and empirical literature on this issue. Unit root test often have low power under general assumptions about the data generating process, so that very long series would be required to be able to reject the unit root hypothesis. One of the objectives of this paper is to show that, while the statistical decision between a random walk and a mean reversion model can be very difficult, the implication of that decision may have enormous consequences for option prices and implied volatilities.

Furthermore, it is now well known that volatility in financial markets is not constant but changes over time, see Schwert (1989) for an early investigation. There are however recent empirical studies of commodity markets and in particular of energy markets showing that volatilities in these markets are generally higher and have even more variation than in financial markets, see e.g. Pindyck (2002) for the oil market and the empirical study of Hafner and Herwartz (2002) for the oil and natural gas market.

The main purpose of this paper is to derive simple formulae that could be used, for example, in spreadsheets to evaluate option prices and implied volatilities, under a model that captures the stylized facts mentioned above. In order to discuss the implications of the assumption about the drift, our basic model framework consists alternatively of a geometric Brownian motion and a mean reversion model. For both cases, volatility is allowed to be stochastic. The main idea to obtain suitable results is to use discrete time approximations, that is, a random walk and an $\mathrm{AR}(1)$ model, respectively, with GARCH errors. Simple solutions are available if second order approximations are used in the spirit of Hull and White (1987). As they note, the higher order terms in the expansion such as skewness and kurtosis are much smaller than the variance term, so that this approximation should be reasonably close for practical purposes. However, Hull and White only consider the random walk case. In a recent paper, Hafner and Herwartz (2001) investigate the effects of stochastic volatility in a mean reversion framework, resorting to Monte Carlo simulations when using discrete time models. In this paper, I use simple approximations to avoid time-consuming simulations. The derived recursive equations could be very useful, for example in spreadsheet implementations.

The remainder of the paper is organized as follows. The following section presents the model framework in continuous time, assuming time-varying but deterministic volatility. Section 3 introduces stochastic volatility and explains how discrete time approximations can be used. Section 4 applies the GARCH model to obtain predictions of the instantaneous volatilities, that in turn are used for predictions of implied volatilities. Finally, Section 5 presents an empirical application to a European option on the electricity spot price of the Scandinavian electricity market NordPool. It turns out that implied volatility 
forecasts of a random walk and an AR(1) model differ substantially, even though the estimated AR(1) coefficient is close to one. Finally, Section 6 concludes and gives an outlook to future work.

\section{Two basic models}

The model framework of our paper consists of two basic model types, the one being a geometric Brownian motion (GBM) commonly used in financial markets, the other a mean-reverting (MR) process that is more common in commodity markets. To capture deterministic calendar effects and seasonality, we will in both cases allow for time-varying mean and variance coefficients. Denote by $X_{t}$ the spot price of the underlying process. In this paper, we will consider the pricing of a European call option, whose payoff at the exercise date $T$ is given by $C_{T}=\max \left(0, X_{T}-K\right)$, where the strike price $K$ is a fixed parameter. The pricing of European put options with payoff $P_{T}=\max \left(0, K-X_{T}\right)$ follows analogously.

\subsection{Geometric Brownian Motion}

The geometric Brownian motion (GBM) model has a large tradition in explaining prices at equity and foreign exchange markets, because of its link with the efficient market hypothesis. It basically assumes that returns should be unpredictable. Let us consider a general version of this model allowing for seasonality which can be written as

$$
d X_{t}=\mu_{t} X_{t} d t+\sigma_{t} X_{t} d W_{t}
$$

where $W_{t}$ is a standard Wiener process and $\mu_{t}$ and $\sigma_{t}$ are deterministic functions of time and may take into account seasonal effects in the mean and variance of the process. By Itô's lemma, we obtain

$$
d \log X_{t}=\left(\mu_{t}-\frac{1}{2} \sigma_{t}^{2}\right) d t+\sigma_{t} d W_{t}
$$

and integrating both sides yields

$$
\log X_{t}=\log X_{0}+\int_{0}^{t}\left(\mu_{s}-\frac{1}{2} \sigma_{s}^{2}\right) d s+\int_{0}^{t} \sigma_{s} d W_{s}
$$

We see that the conditional distribution of $\log X_{t}$ is normal with mean and variance given by

$$
\mathrm{E}_{0}\left[\log X_{t}\right]=\log X_{0}+\int_{0}^{t}\left(\mu_{s}-\frac{1}{2} \sigma_{s}^{2}\right) d s
$$


and

$$
\operatorname{Var}_{0}\left(\log X_{t}\right)=\int_{0}^{t} \sigma_{s}^{2} d s
$$

The conditional distribution of $X_{t}$ is therefore lognormal ${ }^{1}$ with mean

$$
\mathrm{E}_{0}\left[X_{t}\right]=X_{0} e^{\int_{0}^{t} \mu_{s} d s}
$$

and variance

$$
\operatorname{Var}_{0}\left(X_{t}\right)=X_{0}^{2} e^{2 \int_{0}^{t} \mu_{s} d s}\left(e^{\int_{0}^{t} \sigma_{s}^{2} d s}-1\right)
$$

The Black and Scholes (1973) framework assumes that $\mu_{t}=\mu$ and $\sigma_{t}=\sigma$ are constant parameters, in which case the no-arbitrage price for a European call option is given by

$$
C_{B S, t}\left(\sigma^{2}\right)=X_{t} \Phi\left(d_{1}\right)-K e^{-r(T-t)} \Phi\left(d_{2}\right)
$$

with $\Phi$ denoting the standard normal distribution function,

$$
d_{1}\left(\sigma^{2}\right)=\frac{\log \left(X_{t} / K\right)+\left(r+\sigma^{2} / 2\right)(T-t)}{\sigma \sqrt{T-t}},
$$

where $r$ is the constant risk-free interest rate and $d_{2}=d_{1}-\sigma \sqrt{T-t}$. The main argument of Black and Scholes (1973) in deriving this formula was that it is possible to construct a hedge portfolio only using the underlying and bonds that eliminates the risk of the call option.

In the general case with deterministic functions $\mu_{t}$ and $\sigma_{t}$, prices remain log-normally distributed and it is still possible to construct a riskless hedge portfolio only using the underlying and bonds. Thus, the Black-Scholes formula remains valid. Since the drift function does not enter in (4), the only change appears in the volatility. Note however that the drift function has an influence on the estimated volatility and therefore on the option price. Let us define the average volatility until maturity as

$$
V_{t, T}=\frac{1}{T-t} \operatorname{Var}_{t}\left(\log X_{T}\right)
$$

which in the GBM case is given by

$$
V_{t, T}=\frac{1}{T-t} \int_{t}^{T} \sigma_{s}^{2} d s .
$$

Thus, $V_{t, T}$ is an average of instantaneous volatilities $\sigma_{s}^{2}, t \leq s \leq T$. The no-arbitrage call option price is then given by

$$
C_{t}=C_{B S, t}\left(V_{t, T}\right)
$$

\footnotetext{
${ }^{1}$ If $X \sim N\left(\mu, \sigma^{2}\right)$, then $e^{X}$ is lognormal with mean $e^{\mu+\sigma^{2} / 2}$ and variance $e^{2 \mu+\sigma^{2}}\left(e^{\sigma^{2}}-1\right)$
} 
Equation (8) says that the fair option value is obtained by plugging the value of $V_{t, T}$ into the Black and Scholes formula. Thus, $V_{t, T}$ can be interpreted as the so-called implied volatility parameter of the model, as opposed to the implied volatility parameter of the market that, when plugged into the Black and Scholes formula returns an observed market price. The GBM model implied volatility, $V_{t, T}$, is therefore an equally weighted average of future instantaneous volatilities until the expiry date of the option.

Traders sometimes look at historical volatilities, i.e., standard deviations of past returns over some moving window of fixed size, and compare them to implied volatilities of traded options to see whether they are over- or undervalued. The preceding paragraph makes clear that in general this is not the appropriate procedure, because implied volatilities are averages of future instantaneous volatilities, not historic ones, and of course they can be quite different. This is even more so if prices are mean-reverting, the case that will be discussed next.

\subsection{Mean reversion}

The basic idea of the mean reversion model is that prices fluctuate around a deterministic trend, and the higher the deviation from this trend, the stronger prices are pulled back to the trend. This model is given by

$$
d \log X_{t}=\kappa\left(\mu_{t}-\log X_{t}\right) d t+\sigma_{t} d W_{t}
$$

where the deterministic trend, $\mu_{t}$, is allowed to vary with time. The speed of mean reversion is determined by the parameter $\kappa>0$. An explicit solution can be found to be

$$
\log X_{t}=e^{-\kappa t} \log X_{0}+\kappa \int_{0}^{t} e^{-\kappa(t-s)} \mu_{s} d s+\int_{0}^{t} e^{-\kappa(t-s)} \sigma_{s} d W_{s} .
$$

Again, the conditional distribution of $\log X_{t}$ is normal, but now with mean and variance given by

$$
\mathrm{E}_{0}\left[\log X_{t}\right]=e^{-\kappa t} \log X_{0}+\kappa \int_{0}^{t} e^{-\kappa(t-s)} \mu_{s} d s
$$

and

$$
\operatorname{Var}_{0}\left(\log X_{t}\right)=\int_{0}^{t} e^{-2 \kappa(t-s)} \sigma_{s}^{2} d s
$$

Consequently, the call option price is still given by (8) but now with

$$
V_{t, T}=\frac{1}{T-t} \int_{t}^{T} e^{-2 \kappa(T-s)} \sigma_{s}^{2} d s
$$


Thus, $V_{t, T}$ is a weighted average of instantaneous volatilities $\sigma_{s}^{2}, t \leq s \leq T$, where the weights are exponentially declining with the time to maturity $T-s$. Interpreting $V_{t, T}$ again as the implied volatility, we see that the implied volatility in the MR model is dominated by instantaneous volatilities close to the expiry date of the option. The explanation is quite obvious: If prices are mean-reverting, initial shocks die out quickly because a large deviation from the drift function tends to be followed by subsequent movements in the opposite direction. Close to maturity, on the other hand, the mean reversion rate may not be strong enough to offset large shocks until the option expires. In other words, large instantaneous volatilities may not have much effect on the implied volatility of the option if the time to maturity is still large and the mean reversion rate is not too small.

\section{Stochastic volatility}

The results of the preceding section are valid only for deterministic volatility. In many empirical studies in financial or commodity markets, the random character of volatility has been demonstrated. Although alternative models have been established, there is common agreement that volatility is mean reverting or stationary. A suitable model in continuous time could be formulated as

$$
d \sigma_{t}^{2}=\left(\omega_{t}-\theta \sigma_{t}^{2}\right) d t+\delta \sigma_{t}^{2} d Z_{t}
$$

where $Z_{t}$ is a standard Wiener process independent of $W_{t}, \theta$ and $\delta$ are constant parameters, and $\omega_{t}$ is a deterministic function of time that may take into account seasonality in variance. Note that (11) is more general than the model employed by Hull and White (1987), who set $\omega_{t}=0$ which implies a less realistic non-stationary volatility process. Also, (11) is appealing because it is the diffusion limit of the popular discrete time $\operatorname{GARCH}(1,1)$ model, as shown by Nelson (1990).

Under stochastic instantaneous volatility $\sigma_{s}^{2}$ and given information at time $t$, the average volatility over the option's lifetime, $V_{t, T}$, is no longer deterministic but random. The analytic form of the distribution of $V_{t, T}$ may not be known. Moreover, because the stochastic differential equation for $\log X_{t}$ now involves a mixture of two independent Wiener processes, the conditional distribution of $\log X_{t}$ is no longer Gaussian but fattailed. While this is closer to empirical observations, it renders option pricing more difficult since the BS formula cannot be used directly.

What is also needed is an assumption on the risk premium of volatility. Hull and White (1987) show that, assuming a volatility risk premium of zero, the option price is 
given by

$$
C_{t}=E_{t}\left[C_{B S}\left(V_{t, T}\right)\right]
$$

where the expectation is taken with respect to the (unknown) distribution of $V_{t, T}$. Even though the distribution is unknown, its lower moments may be easily calculated. Therefore, a second order Taylor approximation is feasible and given by

$$
C_{t} \approx C_{B S, t}\left(\bar{V}_{t, T}\right)+\frac{1}{2} \frac{\partial^{2} C_{B S, t}}{\partial V_{t, T}^{2}}\left(\bar{V}_{t, T}\right) \operatorname{Var}_{t}\left(V_{t, T}\right)
$$

where $\bar{V}_{t, T}=\mathrm{E}_{t}\left[V_{t, T}\right]$ is the expected average volatility over the option's lifetime. The second derivative of the BS call price formula with respect to volatility, evaluated at $\bar{V}_{t, T}$, is easily calculated to be

$$
C^{\prime \prime}=\frac{\partial^{2} C_{B S, t}}{\partial V_{t, T}^{2}}\left(\bar{V}_{t, T}\right)=\frac{X_{t} \psi\left(d_{1}\right)\left(d_{1} d_{2}-1\right) \sqrt{T-t}}{4 \bar{V}_{t, T}^{3 / 2}}
$$

where $d_{1}=d_{1}\left(\bar{V}_{t, T}\right)$ is given in (5), $d_{2}=d_{1}\left(\bar{V}_{t, T}\right)-\sqrt{V_{t, T}(T-t)}$, and $\psi$ is the standard normal density function.

Looking closer at $(13)$ it can be seen that 'at-the-money' (i.e. $X_{t} \approx K$ ), the second derivative in (13) is negative and therefore $C_{t}<C_{B S, t}$, whereas in- or out-of-the money it is positive, and hence $C_{t}>C_{B S, t}$. This produces the well-known 'smile' in implied volatilities, i.e., option prices are smaller at-the-money and larger in- and out-of-themoney compared to a model with constant volatility. The smile is, however, usually expressed in terms of the implied volatility, $V_{t, T}^{I}$, which is implicitly defined by $C_{t}=$ $C_{B S, t}\left(V_{t, T}^{I}\right)$. One can not solve for $V_{t, T}^{I}$ analytically, but simple numerical search algorithms can be used. To get some more intuition, let us do a second order Taylor expansion for $V_{t, T}^{I}$, i.e.

$$
C_{B S, t}\left(V_{t, T}^{I}\right)=C_{B S, t}\left(\bar{V}_{t, T}\right)+\left(V_{t, T}^{I}-\bar{V}_{t, T}\right) C^{\prime}+\frac{1}{2}\left(V_{t, T}^{I}-\bar{V}_{t, T}\right)^{2} C^{\prime \prime}
$$

where $C^{\prime \prime}$ is given by (13), and

$$
C^{\prime}=\frac{\partial C_{B S, t}}{\partial V_{t, T}}\left(\bar{V}_{t, T}\right)=\frac{X_{t} \psi\left(d_{1}\right) \sqrt{T-t}}{2 \bar{V}_{t, T}^{1 / 2}}
$$

Note that (15) is not identical to the usually called Vega, which is the derivative with respect to the standard deviation, not the variance. Combining (14) with (12), we obtain the following quadratic equation for $Z=V_{t, T}^{I}-\bar{V}_{t, T}$,

$$
\frac{1}{2} C^{\prime \prime} Z^{2}+C^{\prime} Z-\frac{1}{2} C^{\prime \prime} \operatorname{Var}\left(V_{t, T}\right)=0,
$$


which for $C^{\prime \prime} \neq 0$ gives the following solution.

$$
V_{t, T}^{I}=\bar{V}_{t, T}-\frac{C^{\prime}}{C^{\prime \prime}}+\operatorname{sign}\left(C^{\prime \prime}\right) \sqrt{\left(\frac{C^{\prime}}{C^{\prime \prime}}\right)^{2}+\operatorname{Var}_{t}\left(V_{t, T}\right)} .
$$

Note that the term $C^{\prime} / C^{\prime \prime}$ takes the simple expression $2 \bar{V}_{t, T} /\left(d_{1} d_{2}-1\right)$. Now, if $\operatorname{Var}_{t}\left(V_{t, T}\right)$ is small, then $V_{t, T}^{I} \approx \bar{V}_{t, T}$, so the implied volatility is close to the mean of $V_{t, T}$. On the other hand, if $\operatorname{Var}_{t}\left(V_{t, T}\right)$ is large, then we have to distinguish two cases: If $C^{\prime \prime}<0$ (at-themoney), then $V_{t, T}^{I}<\bar{V}_{t, T}$, whereas if $C^{\prime \prime}>0$ (in- or out-of-the-money), then $V_{t, T}^{I}>\bar{V}_{t, T}$. This is the famous smile effect, and the amplitude of the smile is determined by the magnitude of $\operatorname{Var}\left(V_{t, T}\right)$.

Let us now consider the situation in practice where one has $t$ observations in discrete time, $X_{1}, \ldots, X_{t}$ and corresponding estimates of instantaneous volatility $\sigma_{1}^{2}, \ldots, \sigma_{t}^{2}$. Based on this information at time $t$, one can build forecasts of future instantaneous volatilities $\sigma_{t+1}^{2}, \ldots, \sigma_{T}^{2}$, and we will see in the next section how this can be achieved in GARCH models. If the frequency of observations is sufficiently high, which is usually the case when one has, say, daily data, we can use the following simple discrete time approximation to the diffusion models:

$$
\log X_{t} / X_{t-1}=\mu_{t}+\varepsilon_{t}
$$

and

$$
\log X_{t}=\phi \log X_{t-1}+\kappa \mu_{t}+\varepsilon_{t}
$$

where $\phi=e^{-\kappa}$, and $\varepsilon_{t}$ is an error term that, conditional on the information set at time $t-1$, has a normal distribution with mean zero and variance $\sigma_{t}^{2}$. The discrete time models in (18) and (19) are the well known random walk with drift and AR(1) model, respectively, both having heteroskedastic error terms. These models can be estimated using any standard statistical software, and the parameter estimates of the continuous time models are immediately obtained.

In order to evaluate the option price in (12), we need to calculate the conditional variance of $V_{t, T}$, given in (7) and (10), respectively. Using our discrete time approximation, we can replace the integral contained in the expression for $V_{t, T}$ by a sum to get

$$
V_{t, T}=\frac{1}{T-t} \sum_{i=1}^{T-t} \sigma_{t+i}^{2}
$$

and

$$
V_{t, T}=\frac{1}{T-t} \sum_{i=1}^{T-t} \phi^{2(T-t-i)} \sigma_{t+i}^{2}
$$


in the GBM and MR model, respectively. As for the mean and variance of $V_{t, T}$, we obtain

$$
\begin{aligned}
\bar{V}_{t, T} & =\frac{1}{T-t} \sum_{i=1}^{T-t} \mathrm{E}_{t}\left[\sigma_{t+i}^{2}\right] \\
\operatorname{Var}_{t}\left(V_{t, T}\right) & =\frac{1}{(T-t)^{2}}\left\{\sum_{i=1}^{T-t} \operatorname{Var}_{t}\left(\sigma_{t+i}^{2}\right)+2 \sum_{i<j}^{T-t} \operatorname{Cov}_{t}\left(\sigma_{t+i}^{2}, \sigma_{t+j}^{2}\right)\right\}
\end{aligned}
$$

and

$$
\begin{aligned}
\bar{V}_{t, T} & =\frac{1}{T-t} \sum_{i=1}^{T-t} \phi^{2(T-t-i)} \mathrm{E}_{t}\left[\sigma_{t+i}^{2}\right] \\
\operatorname{Var}_{t}\left(V_{t, T}\right) & =\frac{1}{(T-t)^{2}}\left\{\sum_{i=1}^{T-t} \phi^{4(T-t-i)} \operatorname{Var}_{t}\left(\sigma_{t+i}^{2}\right)\right. \\
& \left.+2 \sum_{i<j}^{T-t} \phi^{2(T-t-i)+2(T-t-j)} \operatorname{Cov}_{t}\left(\sigma_{t+i}^{2}, \sigma_{t+j}^{2}\right)\right\}
\end{aligned}
$$

respectively. To calculate $\bar{V}_{t, T}$, all we need are predictions of the instantaneous volatilities, $\mathrm{E}_{t}\left[\sigma_{t+i}^{2}\right]$. The variance of $V_{t, T}$ depends on all variances and covariances of instantaneous volatilities between $t$ and $T$. Note that, by definition,

$$
\begin{aligned}
\operatorname{Var}_{t}\left(\sigma_{t+i}^{2}\right) & =\mathrm{E}_{t}\left[\sigma_{t+i}^{4}\right]-\mathrm{E}_{t}\left[\sigma_{t+i}^{2}\right]^{2} \\
\operatorname{Cov}_{t}\left(\sigma_{t+i}^{2}, \sigma_{t+j}^{2}\right) & =\mathrm{E}_{t}\left[\sigma_{t+i}^{2} \sigma_{t+j}^{2}\right]-\mathrm{E}_{t}\left[\sigma_{t+i}^{2}\right] \mathrm{E}_{t}\left[\sigma_{t+j}^{2}\right]
\end{aligned}
$$

so that one needs to calculate the conditional expectations $\mathrm{E}_{t}\left[\sigma_{t+i}^{4}\right]$ and $\mathrm{E}_{t}\left[\sigma_{t+i}^{2} \sigma_{t+j}^{2}\right]$. As we will see in the next section, computationally convenient recursive formulae can be applied if one uses the popular GARCH model class.

\section{Using GARCH approximations}

We now look at ways to conveniently compute the mean and variance of $V_{t, T}$ that is required for the second order approximation in (12). Perhaps the most popular discrete time volatility model is the so-called GARCH model by Bollerslev (1986) that is widely used in practice. For a survey on theory and estimation see, e.g., Bollerslev, Engle and Nelson (1994).

Consider the error terms $\varepsilon_{t}$ in (18) and (19), respectively. The often used GARCH(1,1) model then specifies $\operatorname{Var}_{t-1}\left(\varepsilon_{t}\right)=\sigma_{t}^{2}$ with

$$
\sigma_{t}^{2}=\omega+\alpha \varepsilon_{t-1}^{2}+\beta \sigma_{t-1}^{2}
$$


where $\omega, \alpha$, and $\beta$ are positive parameters. One of the features of this specification is that it allows for convenient prediction of future volatilities, because the $i$-step conditional mean is given by

$$
\mathrm{E}_{t}\left[\sigma_{t+i}^{2}\right]=\omega \frac{1-\delta^{i-1}}{1-\delta}+\delta^{i-1} \sigma_{t+1}^{2}
$$

where $\delta=\alpha+\beta$. This converges to the unconditional instantaneous volatility $\sigma^{2}$ if $\delta<1$, where $\sigma^{2}$ is then given by

$$
\sigma^{2}=\lim _{i \rightarrow \infty} \mathrm{E}_{t}\left[\sigma_{t+i}^{2}\right]=\frac{\omega}{1-\delta}
$$

One can now extend the standard GARCH model in (24) to allow for seasonality. A model that corresponds to the diffusion model (11) would be

$$
\sigma_{t}^{2}=\omega_{t}+\alpha \varepsilon_{t-1}^{2}+\beta \sigma_{t-1}^{2}
$$

where $\omega_{t}$ is a deterministic function of time. In this model, seasonality occurs in the level of volatility, whereas the parameters that drive the persistence, $\alpha$ and $\beta$, are assumed to remain constant.

For model (25), simple recursive formulae can be found for the optimal predictors. Then,

$$
\begin{aligned}
\mathrm{E}_{t}\left[\sigma_{t+2}^{2}\right] & =\omega_{t+2}+\delta \sigma_{t+1}^{2} \\
\mathrm{E}_{t}\left[\sigma_{t+i}^{2}\right] & =\omega_{t+i}+\delta \mathrm{E}_{t}\left[\sigma_{t+i-1}^{2}\right], \quad i \geq 3
\end{aligned}
$$

To determine the variance of $V_{t, T}$, we further need the conditional variances and autocovariances of $\sigma_{t+i}^{2}$. Assuming normality of standardized errors, i.e. $\varepsilon_{t} / \sigma_{t} \sim N(0,1)$, and defining $\gamma=3 \alpha^{2}+\beta^{2}+2 \alpha \beta$, we obtain for the fourth moment predictions,

$$
\begin{aligned}
\mathrm{E}_{t}\left[\sigma_{t+2}^{4}\right] & =\omega_{t+2}^{2}+\gamma \sigma_{t+1}^{4}+2 \delta \omega_{t+2} \sigma_{t+1}^{2} \\
\mathrm{E}_{t}\left[\sigma_{t+i}^{4}\right] & =\omega_{t+i}^{2}+\gamma E_{t}\left[\sigma_{t+i-1}^{4}\right]+2 \delta \omega_{t+i} \mathrm{E}_{t}\left[\sigma_{t+i-1}^{2}\right], \quad i \geq 3 .
\end{aligned}
$$

Note that $\gamma<1$ is required, otherwise fourth moments would not exist and the conditional expectation in (28) would tend to infinity with increasing horizon $i$. The variance terms are now simply given by equation (23). Likewise, it is straightforward to obtain the covariance terms

$$
\operatorname{Cov}_{t}\left[\sigma_{t+i}^{2}, \sigma_{t+j}^{2}\right]=\delta^{j-i} \operatorname{Var}_{t}\left(\sigma_{t+i}^{2}\right), \quad 1 \leq i \leq j .
$$

These covariances are non-negative and, due to the assumed covariance-stationarity of the model $(\delta<1)$, they converge to zero for increasing temporal distance, $j-i$, between the two instantaneous volatilities. 


\section{An application to options on electricity prices}

In this section I give an example of an application to the pricing of European options on the spot price of electricity in the Scandinavian market, called NordPool (see www.nordpool.no). This market is among the most developed electricity markets in Europe and includes trading of many liquid physical and financial contracts. Options are mostly written on futures and forwards, but for illustrative purposes I will consider a (fictitious) European option on the spot price here. The spot market is physical where prices are fixed every day for the 24 hours of the next day. The arithmetic average of the hourly prices is commonly referred to as the daily spot price, which is our underlying price process. In order to simplify the seasonality structure, I discarded the weekend prices. Data are from January 1, 1996, to September 17, 2002, which makes a total of 1751 observations. The sample standard deviation of daily returns is 0.0911 , or annualized 147 $\%$, which is much higher than the typically observed volatilities at financial markets. It is not only due to a few outliers.

I estimate the following simple time series model fitted to the logarithm of the spot price, $\log X_{t}$.

$$
\log X_{t}=c+\beta_{1} \cos \left(\frac{2 \pi}{P} t\right)+\beta_{2} \sin \left(\frac{2 \pi}{P} t\right)+\phi \log X_{t-1}+\varepsilon_{t},
$$

where the period $P$ in the trigonometric terms is fixed to one year. Alternatively, I fix the parameter $\phi$ to unity to estimate the following random walk with drift model:

$$
\log X_{t} / X_{t-1}=c+\beta_{1} \cos \left(\frac{2 \pi}{P} t\right)+\beta_{2} \sin \left(\frac{2 \pi}{P} t\right)+\varepsilon_{t},
$$

For both models, the errors $\varepsilon_{t}$ have conditional variance $\sigma_{t}^{2}$, which is specified as

$$
\sigma_{t}^{2}=\omega+\gamma_{1} \cos \left(\frac{2 \pi}{P} t\right)+\gamma_{2} \sin \left(\frac{2 \pi}{P} t\right)+\alpha \varepsilon_{t-1}^{2} .
$$

This can be considered as an $\mathrm{ARCH}(1)$ model with seasonality. Neither in the conditional mean nor in the conditional variance were higher order lags found to be significant. Maximum likelihood estimation yields the results reported in Table 1. The maximum likelihood values are 1985.2 for the random walk model and 1995.7 for the mean reverting value, indicating a superior fit of the latter.

For both estimated models, I calculate the prices of at-the-money European call options for alternative times to maturity using the information at the end of the sample, September 17, 2002, and using equation (12). Then, implied volatilities are obtained by equation (17) (numerical search algorithms yield very close results). Figure 1 shows the 


\begin{tabular}{ccccr}
\hline \hline & \multicolumn{2}{c}{ MR } & \multicolumn{2}{c}{ Random Walk $(\phi=1)$} \\
\hline$c$ & 0.105838 & $(4.01)$ & 0.001780 & $(1.03)$ \\
$\beta_{1}$ & 0.004785 & $(1.48)$ & 0.001852 & $(0.93)$ \\
$\beta_{2}$ & -0.005131 & $(-2.13)$ & -0.004947 & $(-1.90)$ \\
$\phi$ & 0.979299 & $(186.8)$ & & \\
\hline$\omega$ & 0.004697 & $(4.27)$ & 0.004707 & $(59.09)$ \\
$\gamma_{1}$ & 0.000710 & $(0.58)$ & 0.000793 & $(7.44)$ \\
$\gamma_{2}$ & 0.002337 & $(2.41)$ & 0.002080 & $(15.55)$ \\
$\alpha$ & 0.443185 & $(4.04)$ & 0.446952 & $(13.47)$ \\
\hline \hline
\end{tabular}

Table 1: Parameter estimates of the models in (29), (30) and (31), applied to the logarithm of the spot price of the Scandinavian electricity market, NordPool, January 1996 to September 2002. T-statistics based on heteroskedasticity-consistent standard errors are reported in parentheses.

implied volatilities for both models as a function of the time to maturity. It becomes obvious that, even though the statistical difference between both models does not seem big, there is a large difference of implied volatilities in particular for increasing maturities. Additional to the implied volatilities, the figure shows the forecast of the instantaneous volatility for the MR model, $\mathrm{E}_{t}\left[\sigma_{t+i}^{2}\right]$ as given by equation (26). The corresponding forecast of the random walk model is very similar, so it is not shown. Note that the mean of instantaneous volatility predictions over one year is close to the empirical mean of $147 \%$, as expected.

The interpretation of the implied volatilities of the random walk model as equally weighted averages of instantaneous volatilities becomes obvious from the graph. On the other hand, the implied volatilities of the mean reversion model decline with time to maturity. This reflects the different weighting scheme, as can be best seen in the equations for $\bar{V}_{t, T}$ in (20) and (21). It is remarkable that even an estimate of $\phi$ so close to one has such an enormous effect for the implied volatility predictions.

The effect of stochastic volatility in this example is found to be important only for short maturity options. The reason is that the value of $\operatorname{Var}_{t}\left(V_{t, T}\right)$ in equation (17) is $13 \%$ of the value of $\left(C^{\prime} / C^{\prime \prime}\right)^{2}$ for one day to maturity, $5 \%$ for two days, $2 \%$ for three days, and then decreases further, so that for long maturity options the implied volatility is close to the mean of $V_{t, T}$. This is certainly due to the fact that there is only an ARCH model of order one in our model. If higher order terms were found to be significant in a general 


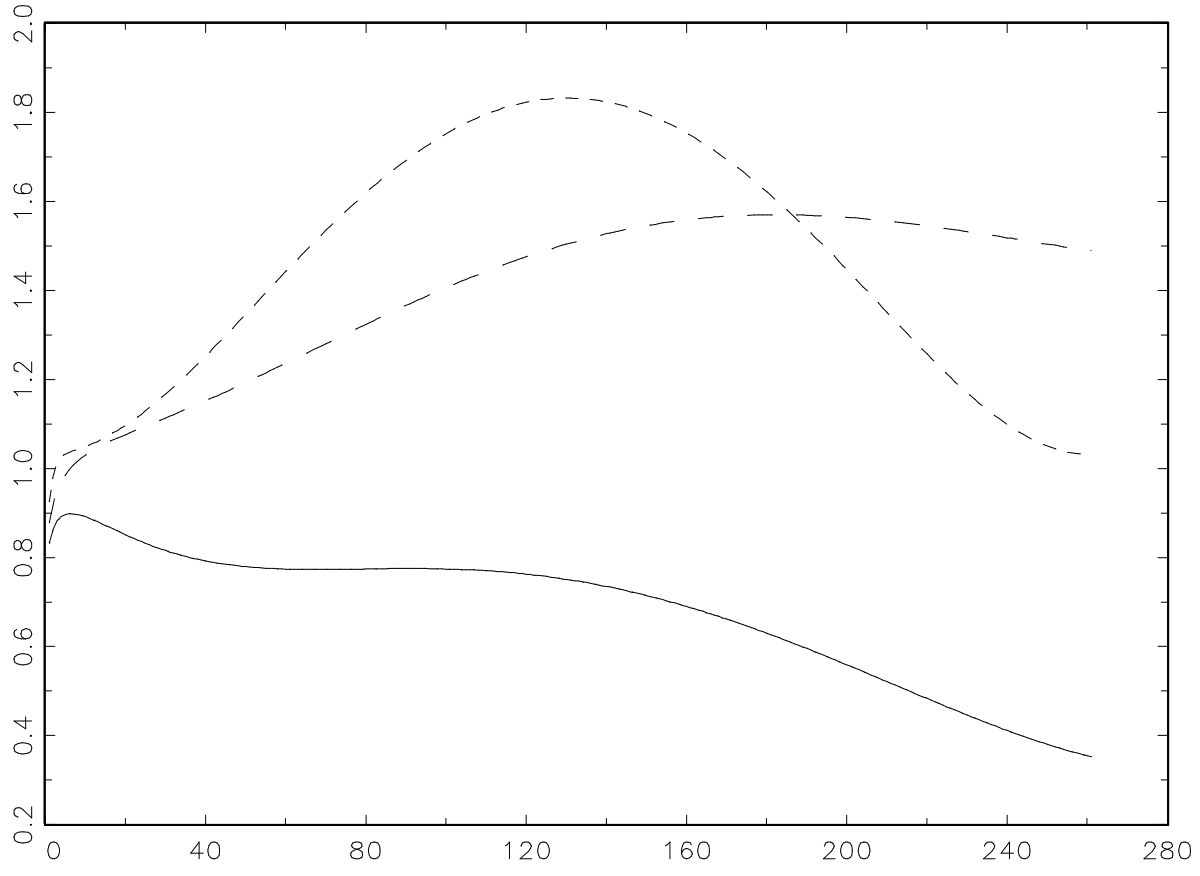

Figure 1: Predictions of implied volatilities under the random walk model (long dashed line) and the mean reversion model (solid line) as a function of the trading days to maturity. The short dashed line is the prediction of instantaneous volatility (using the mean reversion model, but very similar under the random walk model). The considered derivative is a European call option on the spot price of the Scandinavian electricity market, NordPool. The current date of the option is chosen to be the end of the sample, i.e., September 17, 2002. 
$\operatorname{GARCH}(p, q)$ model, then stochastic volatility would have a stronger effect on implied volatilities with long maturities.

\section{Conclusions and Outlook}

In this paper, I motivate the use of spreadsheets as a convenient platform to evaluate derivatives, even under complicated situations such as stochastic volatility. Suitable approximations can be found that have the advantage of immediate availability of results. Formulae are provided for a random walk and a mean reversion model with time-varying drift and volatility. For the stochastic volatility case, I suggest to use GARCH approximations that are frequently used in practice. The formulae provided in this paper could be easily extended to more general GARCH models such as threshold GARCH models, to take into account asymmetry of the progation of noise in the volatility equation.

For the application to options on electricity prices, it appeared that stochastic volatility is important. However, it mattered even more for predictions of implied volatilities whether a mean reversion or random walk model was specified, although the estimated AR(1) parameter was close to one. This result should be a warning against an uncritical use of a random walk model when there is some evidence of mean reversion. A consequence of a false random walk assumption could be a strong overvaluation of implied volatilities, as shown in the example. Of course, the statistical decision between the two models is often difficult and unit root tests are not always giving reliable answers. Especially in commodity and energy markets, there are often more economic arguments for mean reversion in prices than there is for random walk, as opposed to financial markets. Further empirical evidence on derivatives in commodity markets may help to clarify how practitioners evaluate implied volatilities.

\section{References}

Aydınlı, G. (2002), Net based spreadsheets in quantitative finance, in: Applied Quantitative Finance.

Aydınl, G., Härdle, W., Kleinow, T. \& Sofyan, H. (2001), ReX: Linking XploRe to Excel, ISM Symposium 'Statistical Software in the Internet Age', February 19-21.

Bachelier, L. (1900), Théorie de la spéculation, Annales de l'Ecole Normale Superieure $17,21-86$. 
Black, F. and M. Scholes (1973), The pricing of options and corporate liabilities, Journal of Political Economy 81, 637-659.

Bollerslev, T. (1986), Generalized autoregressive conditional heteroskedasticity, Journal of Econometrics 31, 307-327.

Bollerslev, T., Engle, R.F. and Nelson, D.B. (1994), GARCH Models, in: Engle, R.F., \& McFadden, D.L. (eds.) Handbook of Econometrics, Vol. 4, Elsevier, Amsterdam, 2961-3038.

Campbell, J.Y., Lo, A.W., \& MacKinlay, A.C. (1997), The Econometrics of Financial Markets, Princeton.

Cox, J. C., Ingersoll, Jr., J. E. \& Ross, S. A. (1985), A theory of the term structure of interest rates, Econometrica 53, 385-407.

Hafner, C.M. \& Herwartz, H. (2001), Option pricing under linear autoregressive dynamics, heteroskedasticity, and conditional leptokurtosis, Journal of Empirical Finance $8,1-34$.

Hafner, C.M. \& Herwartz, H. (2002), Testing for vectorautoregressive dynamics under heteroskedasticity, Econometric Institute Report 36, Erasmus Universtity Rotterdam.

Hull, J. \& White, A. (1987), The pricing of options on assets with stochastic volatilities, Journal of Finance 42, 281-299.

Nelson, D. B. (1990), ARCH models as diffusion approximations, Journal of Econometrics $45,7-38$.

Pindyck, R.S. (2002), Volatility and commodity price dynamics, M.I.T. working paper, Cambridge, MA.

Schwartz, E. (1997), The stochastic behavior of commodity prices: Implications for valuation and hedging, Journal of Finance 52, 923-973.

Schwert, G. W. (1989), Why does stock market volatility change over time?, Journal of Finance 44, 1115-1153. 